\title{
Random field Ising chains with synchronous dynamics
}

\author{
N S Skantzos and A C C Coolen \\ Department of Mathematics, King's College London, Strand, London WC2R 2LS, UK
}

Received 11 October 1999

\begin{abstract}
We present an exact solution of the one-dimensional random field Ising model (RFIM) with synchronous rather than sequential spin-dynamics, whose equilibrium state is characterized by a temperature-dependent pseudo-Hamiltonian, based upon a suitable adaptation of the techniques originally developed for the sequential (Glauber) dynamics RFIM. Although deriving the solution is somewhat more involved in the present model than in the case of the sequential RFIM, we are able to prove rigorously that the physics of the two RFIM versions are asymptotically identical. We thus recover the familiar devil's staircase form for the integrated density of local magnetizations, and find a non-zero ground state entropy with an infinite number of singularities as a function of the random field strength.
\end{abstract}

\section{Introduction}

The one-dimensional random field Ising model is one of the most extensively studied disordered systems [1-10]. Most of the exact solutions that have been presented rely on a method which goes back to [1]: conditioning the partition function $\mathcal{Z}_{N}$ of an $N$-spin chain on the state $\sigma_{N}$ of the last spin (giving the two quantities $\mathcal{Z}_{N, \pm 1}$ ), and subsequently constructing a recurrence relation expressing the $\mathcal{Z}_{N+1, \pm 1}$ in terms of the $\mathcal{Z}_{N, \pm 1}$. The free energy of the system can be written as an integral over the distribution of the subsequent ratios $k_{N}$ of the conditioned partition functions $\mathcal{Z}_{N, \pm 1}$. Adding new spins to the chain one by one (and thus also new random fields) defines a discrete Markovian process for these ratios, or their probability density $P_{N}(k)$, whose stationary state $P_{\infty}(k)$ fully determines the asymptotic free energy per spin. In early papers based on this technique, approximations of the stationary solution were calculated in certain parameter regimes, e.g. for zero temperature [2]. It was later shown [3] that in certain regions of parameter space, including $T>0$, the integrated stationary density $\hat{P}(k)=\int_{0}^{k} \mathrm{~d} z P(z)$ acquires the form of a highly non-analytic object known as the devil's staircase. The support of the density $P(k)$ is now known to be a zero-measure Cantor set $[8,9,11]$, and the spectrum $f(\alpha)$ of generalized dimensions has been fully calculated $[12,13]$. Phase transitions in the usual thermodynamic sense are absent, but the transition of the fractal dimension of the support of $P(k)$ to $D_{F}<1$ has significant physical consequences; in particular, there are regions in the phase diagram where observables such as the local magnetization take values only from a disconnected set.

In this paper we study an alternative to the more standard types of one-dimensional RFIM models. Here the stochastic microscopic alignment of spins to local fields is not executed sequentially (according to a Glauber rule, leading to the conventional Boltzmanntype equilibrium distribution with the standard Ising Hamiltonian), but the spin states are updated synchronously, i.e. in parallel, at discrete time steps. Ising models with synchronous 
dynamics have been studied, e.g., in the context of neural networks $[14,15]$ and probabilistic cellular automata (see, e.g., [16]). Synchronous execution of the (originally sequential) Glauber laws is known to lead again to a unique equilibrium state [15], which can formally be written in the Boltzmann form, and which in principle enables equilibrium statistical mechanical calculations. However, the parallel dynamics (pseudo-)Hamiltonian is a function of temperature, and thermodynamic relations will generally have to be modified. What is known about the relation between the equilibrium physics of the two types of dynamics concerns mostly infinite-range models. To be specific: in the case of predominantly negative exchange interactions the two dynamics types clearly lead to completely different physics, with periodtwo limit cycles appearing in the synchronous dynamics case. In the case of predominantly positive exchange interactions, however, the picture is less clear. For instance, the phase diagram of the Sherrington-Kirkpatrick [17] model (with $J_{0} \geqslant 0$ ) appears not to be affected by synchronous dynamics [18], whereas for the Hopfield model [19] the phase diagram does change [20]. We are not aware of equilibrium studies involving disordered spin chains with synchronous dynamics.

In solving the parallel dynamics RFIM we find that the standard RFIM techniques need to be adapted, in that conditioning of the partition function on the state of the last spin in the chain is no longer sufficient. The present model involves a more complicated stochastic process in terms of three ratios of conditioned partition functions rather than one. We then show that asymptotically the expectation values of the local magnetizations of sequential and parallel dynamics become identical. We examine the occurrence and properties of the devil's staircase shapes for the integrated probability densities of the relevant observables, and we calculate the ground state entropy, which exhibits the non-trivial behaviour as a function of the random field strength which had also been observed for sequential dynamics chains [5].

\section{Model definitions}

Our model is defined as a collection of $N$ Ising spins $\sigma=\left(\sigma_{1}, \ldots, \sigma_{N}\right) \in\{-1,1\}^{N}$, arranged in a one-dimensional chain. The dynamics is a stochastic alignment to local fields of the familiar form, however, in contrast to the more conventional Glauber-type rules where individual spin updates are made sequentially, here the individual spin updates are made in a fully synchronous way at each discrete time step:

$$
\begin{aligned}
& \forall i \in\{1, \ldots, N\}: \quad \operatorname{Prob}\left[\sigma_{i}(t+1)= \pm 1\right]=\frac{1}{2}\left[1 \pm \tanh \left[\beta h_{i}(\sigma(t))\right]\right] \\
& h_{i}(\sigma(t))=\sum_{j=1}^{N} J_{i j} \sigma_{j}(t)+\theta_{i} .
\end{aligned}
$$

The parameter $\beta=1 / T$ controls the amount of stochasticity in the dynamics; for $\beta=\infty$ the process (1) is a deterministic map, for $\beta=0$ is a fully random map. The variables $J_{i j}$ and $\theta_{i}$ represent spin interactions and external fields, respectively. The Markov chain (1) can alternatively be defined in terms of the microscopic state probability $p_{t}(\sigma)$ :

$$
p_{t+1}(\boldsymbol{\sigma})=\sum_{\sigma^{\prime}} W\left[\boldsymbol{\sigma} ; \boldsymbol{\sigma}^{\prime}\right] p_{t}\left(\boldsymbol{\sigma}^{\prime}\right) \quad W\left[\boldsymbol{\sigma} ; \boldsymbol{\sigma}^{\prime}\right]=\prod_{i=1}^{N} \frac{\mathrm{e}^{\beta \sigma_{i} h_{i}\left(\boldsymbol{\sigma}^{\prime}\right)}}{2 \cosh \left[\beta h_{i}\left(\boldsymbol{\sigma}^{\prime}\right)\right]} .
$$

For any finite $\beta$ and finite $N$ the process (3) is ergodic and evolves into a unique stationary distribution $p_{\infty}(\boldsymbol{\sigma})$. It can be shown that this is an equilibrium state (obeying detailed balance) if and only if $J_{i j}=J_{j i}$ for all pairs $(i, j)$. In particular, there is no need to exclude selfinteractions, which would have been required to find detailed balance in models with sequential dynamics. In the detailed balance case the corresponding equilibrium state probabilities can 
only formally be written in the Boltzmann form $p_{\infty}(\sigma) \sim \exp \left[-\beta \mathcal{H}_{\beta}(\sigma)\right]$, with the so-called pseudo-Hamiltonian $\dagger \mathcal{H}_{\beta}(\boldsymbol{\sigma})$, which was first derived in [15]:

$$
\mathcal{H}_{\beta}(\boldsymbol{\sigma})=-\frac{1}{\beta} \sum_{i=1}^{N} \log \left[2 \cosh \left(\beta h_{i}(\boldsymbol{\sigma})\right)\right]-\sum_{i=1}^{N} \sigma_{i} \theta_{i} .
$$

Due to the synchronous execution of the alignment dynamics, and the resulting non-Gibbsian equilibrium distribution, conventional thermodynamic relations will generally no longer hold. We can still formally define a free energy per spin as

$$
f_{N}=-\frac{1}{\beta N} \log \sum_{\sigma} \mathrm{e}^{-\beta \mathcal{H}_{\beta}(\sigma)}
$$

which will play a useful role as a generating function for equilibrium averages, but which no longer carries the usual thermodynamic significance.

In this paper we consider the case where the external fields $\theta_{i}$ have been drawn independently at random from a symmetric binary distribution $w(\theta)$ with variance $\tilde{\theta}^{2}$, and we choose spin interactions $J_{i j}$ describing a strictly ferromagnetic $(J>0)$ or strictly antiferromagnetic $(J<0)$ (periodic or open) one-dimensional chain:

$$
w(\theta)=\frac{1}{2} \delta[\theta-\tilde{\theta}]+\frac{1}{2} \delta[\theta+\tilde{\theta}] \quad J_{i j}=J\left(\delta_{j, i+1}+\delta_{j, i-1}\right) .
$$

We can always put $\tilde{\theta} \geqslant 0$. Averages over $w(\theta)$ will be denoted as: $\overline{g(\theta)}=\int \mathrm{d} \theta g(\theta) w(\theta)$. The relevant macroscopic equilibrium observables in this system are the overall magnetization $m$ and the next-time/nearest-neighbour correlation function $a$ :

$$
m=\lim _{N \rightarrow \infty} \frac{1}{N} \sum_{i}\left\langle\sigma_{i}\right\rangle_{\text {eq }} \quad a=\lim _{N \rightarrow \infty} \frac{1}{N} \sum_{i}\left\langle\sigma_{i} \tanh \left[\beta h_{i+1}(\sigma)\right]\right\rangle_{\mathrm{eq}}
$$

in which the equilibrium averages are calculated using the Boltzmann distribution with the pseudo-Hamiltonian (4). Note that $m, a \in[-1,1]$. The order parameter $a$ measures equilibrium state correlations of nearest-neighbour spins probed at different but successive times, which follows from the identity

$$
\left\langle\sigma_{i} \tanh \left[\beta h_{i+1}(\sigma)\right]\right\rangle_{\mathrm{eq}}=\lim _{\tau \rightarrow \infty} \frac{1}{\tau} \int_{0}^{\tau} \mathrm{d} t \sigma_{i}(t) \sigma_{i+1}(t+1) .
$$

The observable $m$ follows as the moment of the joint distribution for random fields and local equilibrium magnetizations:

$$
P^{ \pm}(\mu)=\lim _{N \rightarrow \infty} \frac{1}{N} \sum_{i} \delta_{\theta_{i}, \pm \tilde{\theta}} \delta\left[\mu-\left\langle\sigma_{i}\right\rangle_{\text {eq }}\right]
$$

since $m=\int \mathrm{d} \mu \mu\left[P^{+}(\mu)+P^{-}(\mu)\right]$. Using simple general properties such as $\left\langle\sigma_{i}\right\rangle_{\mathrm{eq}}=$ $\left\langle\tanh \left[\beta h_{i}(\sigma)\right]\right\rangle_{\text {eq }}$, which follows directly from stationarity of (3), we also see that (5) generates

$$
a=-\frac{1}{2} \frac{\partial}{\partial J} f_{N}
$$

\section{Solution}

The three key ingredients which in combination render the present model non-trivial are: (i) the short-range connectivity, (ii) the random external fields, and (iii) the synchronous dynamics leading to a non-Boltzmann equilibrium state. If we replace the synchronous dynamics by a standard sequential Glauber one we recover the standard RFIM [2,3], exhibiting transitions to

$\dagger$ Note that this pseudo-Hamiltonian depends on $\beta$, hence the name. 
non-trivial states where the integrated distribution of local magnetizations acquires the form of a devil's staircase and where $\left\langle\sigma_{i}\right\rangle_{\text {eq }}$ itself takes values from the Cantor set [4]. We solve model (2), (3), (6) using a suitable adaptation of the techniques in [2,3], i.e. by studying the effect on the partition function $\mathcal{Z}_{N}$ of adding one extra spin to the chain (and thus one extra random field), which can be described by a Markovian stochastic map for a finite number of characteristic quantities. In the present model with synchronous dynamics, however, this map is considerably more complicated than that found in $[2,3]$. Due to the repeated occurrence of terms of the form $\cosh \beta\left[J\left(\sigma_{i-1}+\sigma_{i+1}\right)+\theta_{i}\right]$ in the synchronous dynamics partition function, adding one spin induces effects which propagate backward over two sites, rather than just one; thus, in order to ensure a closed Markovian form for the map to be created we need to keep track of the states of the last two spins in the chain.

\subsection{Adaptation of the RFIM techniques}

In order to find the free energy per spin (5) we have to calculate the synchronous dynamics partition function $\mathcal{Z}_{N}=\sum_{\sigma} \mathrm{e}^{-\beta \mathcal{H}_{\beta}(\sigma)}$, with the pseudo-Hamiltonian (4), which for an open chain reads

$$
\mathcal{Z}_{N}=\sum_{\sigma_{1} \ldots \sigma_{N}} \Omega_{1}\left[0, \sigma_{1}, \sigma_{2}\right]\left[\prod_{i=2}^{N-1} \Omega_{i}\left[\sigma_{i-1}, \sigma_{i}, \sigma_{i+1}\right]\right] \Omega_{N}\left[\sigma_{N-1}, \sigma_{N}, 0\right]
$$

where

$$
\Omega_{i}\left[\sigma_{i-1}, \sigma_{i}, \sigma_{i+1}\right]=2 \cosh \beta\left[J\left(\sigma_{i-1}+\sigma_{i+1}\right)+\theta_{i}\right] \mathrm{e}^{\beta \sigma_{i} \theta_{i}} .
$$

We adapt the construction in $[2,3]$ and write for $N>1$ the partition function (10) as the sum of four new quantities, which can be interpreted as conditional partition functions in which the states of the last two spins in the chain are prescribed:

$$
\mathcal{Z}_{N}=\mathcal{Z}_{N, \uparrow \uparrow}+\mathcal{Z}_{N, \uparrow \downarrow}+\mathcal{Z}_{N, \downarrow \uparrow}+\mathcal{Z}_{N, \downarrow}
$$

For $N>2$ they are given by

$$
\begin{aligned}
\left(\begin{array}{l}
\mathcal{Z}_{N, \uparrow \uparrow} \\
\mathcal{Z}_{N, \uparrow \downarrow} \\
\mathcal{Z}_{N, \downarrow \uparrow} \\
\mathcal{Z}_{N, \downarrow \downarrow}
\end{array}\right)= & \sum_{\sigma_{1} \ldots \sigma_{N}} \Omega_{1}\left[0, \sigma_{1}, \sigma_{2}\right]\left[\prod_{i=2}^{N-1} \Omega_{i}\left[\sigma_{i-1}, \sigma_{i}, \sigma_{i+1}\right]\right] \\
& \times \Omega_{N}\left[\sigma_{N-1}, \sigma_{N}, 0\right]\left(\begin{array}{cc}
\delta_{\sigma_{N-1}, 1} & \delta_{\sigma_{N}, 1} \\
\delta_{\sigma_{N-1}, 1} & \delta_{\sigma_{N},-1} \\
\delta_{\sigma_{N-1},-1} & \delta_{\sigma_{N}, 1} \\
\delta_{\sigma_{N-1},-1} & \delta_{\sigma_{N},-1}
\end{array}\right) .
\end{aligned}
$$

For $N=2$ one simply has $\mathcal{Z}_{2, \star \star}=\Omega_{1}[0, \star, \star] \Omega_{2}[\star, \star, 0]$, for all $(\star \star) \in\{(\uparrow \uparrow),(\uparrow \downarrow),(\downarrow \uparrow)$, $(\downarrow \downarrow)\}$. For future use we also mention the following simple identity:

$$
\frac{\mathcal{Z}_{2, \uparrow \downarrow} \mathcal{Z}_{2, \downarrow \uparrow}}{\mathcal{Z}_{2, \uparrow \uparrow} \mathcal{Z}_{2, \downarrow \downarrow}}=\frac{\Omega_{1}[0,1,-1] \Omega_{1}[0,-1,1]}{\Omega_{1}[0,1,1] \Omega_{1}[0,-1,-1]} \frac{\Omega_{2}[1,-1,0] \Omega_{2}[-1,1,0]}{\Omega_{2}[1,1,0] \Omega_{2}[0,-1,-1]}=1
$$

which immediately follows from the above definition of the functions $\Omega_{i}[\ldots]$.

Enlarging the chain from $N$ to $N+1$ spins gives rise to four new conditional partition functions $\left\{\mathcal{Z}_{N+1, \star \star}\right\}$, which can be expressed in terms of the previous $\left\{\mathcal{Z}_{N, \star \star}\right\}$. Note that, in contrast to the situation with the standard random field Ising chains [2,3], here this would not have been possible if we had only conditioned the partition function on the state of the last 
spin $\sigma_{N}$ of the chain, i.e. the $\left\{\mathcal{Z}_{N+1, *}\right\}$ cannot be expressed in terms of the $\left\{\mathcal{Z}_{N, \star}\right\}$. After some simple book-keeping we can derive from (12) the following recurrent relations:

$$
\begin{aligned}
& \left(\begin{array}{l}
\mathcal{Z}_{N+1, \uparrow \uparrow} \\
\mathcal{Z}_{N+1, \uparrow \downarrow}
\end{array}\right)=M^{+}\left[\theta_{N+1}, \theta_{N}\right]\left(\begin{array}{c}
\mathcal{Z}_{N, \uparrow \uparrow} \\
\mathcal{Z}_{N, \downarrow \uparrow}
\end{array}\right) \\
& \left(\begin{array}{l}
\mathcal{Z}_{N+1, \downarrow \uparrow} \\
\mathcal{Z}_{N+1, \downarrow \downarrow}
\end{array}\right)=M^{-}\left[\theta_{N+1}, \theta_{N}\right]\left(\begin{array}{l}
\mathcal{Z}_{N, \uparrow \downarrow} \\
\mathcal{Z}_{N, \downarrow \downarrow}
\end{array}\right)
\end{aligned}
$$

in which the two (random) matrices $\boldsymbol{M}^{ \pm}\left[\theta_{N+1}, \theta_{N}\right]$ are defined via

$$
\boldsymbol{M}^{ \pm}\left[\theta^{\prime}, \theta\right]=2 \cosh \left[\beta\left(\theta^{\prime} \pm J\right)\right]\left(\begin{array}{cc}
\mathrm{e}^{\beta \theta^{\prime}} & 0 \\
0 & \mathrm{e}^{-\beta \theta^{\prime}}
\end{array}\right)\left(\begin{array}{cc}
\frac{\cosh [\beta(\theta+2 J)]}{\cosh [\beta(\theta+J)]} & \frac{\cosh [\beta \theta]}{\cosh [\beta(\theta-J)]} \\
\frac{\cosh [\beta \theta]}{\cosh [\beta(\theta+J)]} & \frac{\cosh [\beta(\theta-2 J)]}{\cosh [\beta(\theta-J)]}
\end{array}\right) .
$$

These then combine to form a four-dimensional stochastic process, generated by $4 \times 4$ random matrices $M\left[\theta_{N+1}, \theta_{N}\right]$, the stationary state of which will lead us to the free energy per spin:

$$
\begin{gathered}
M\left[\theta^{\prime}, \theta\right]=\left(\begin{array}{cccc}
M_{11}^{+}\left[\theta^{\prime}, \theta\right] & 0 & M_{12}^{+}\left[\theta^{\prime}, \theta\right] & 0 \\
M_{21}^{+}\left[\theta^{\prime}, \theta\right] & 0 & M_{22}^{+}\left[\theta^{\prime}, \theta\right] & 0 \\
0 & M_{11}^{-}\left[\theta^{\prime}, \theta\right] & 0 & M_{12}^{-}\left[\theta^{\prime}, \theta\right] \\
0 & M_{21}^{-}\left[\theta^{\prime}, \theta\right] & 0 & M_{22}^{-}\left[\theta^{\prime}, \theta\right]
\end{array}\right) \\
f_{\infty}=-\lim _{N \rightarrow \infty} \frac{1}{\beta N} \log \left\{\left(\begin{array}{l}
1 \\
1 \\
1 \\
1
\end{array}\right) \cdot\left[\prod_{i=2}^{N-1} M\left[\theta_{i+1}, \theta_{i}\right]\right]\left(\begin{array}{l}
\mathcal{Z}_{2, \uparrow \uparrow}\left[\theta_{2}, \theta_{1}\right] \\
\mathcal{Z}_{2, \uparrow \downarrow}\left[\theta_{2}, \theta_{1}\right] \\
\mathcal{Z}_{2, \downarrow \uparrow}\left[\theta_{2}, \theta_{1}\right] \\
\mathcal{Z}_{2, \downarrow \downarrow}\left[\theta_{2}, \theta_{1}\right]
\end{array}\right)\right\} .
\end{gathered}
$$

In order to evaluate (17) it will turn out helpful to define the following three ratios of the conditional partition functions:

$$
k_{n}^{(1)}=\mathrm{e}^{2 \beta \theta_{n}} \frac{\mathcal{Z}_{n, \uparrow \downarrow}}{\mathcal{Z}_{n, \uparrow \uparrow}} \quad k_{n}^{(2)}=\frac{\cosh \left[\beta\left(\theta_{n}+J\right)\right]}{\cosh \left[\beta\left(\theta_{n}-J\right)\right]} \frac{\mathcal{Z}_{n, \downarrow \downarrow}}{\mathcal{Z}_{n, \uparrow \downarrow}} \quad k_{n}^{(3)}=\mathrm{e}^{2 \beta \theta_{n}} \frac{\mathcal{Z}_{n, \downarrow \downarrow}}{\mathcal{Z}_{n, \downarrow \uparrow}} .
$$

From the recurrent relations (14) it follows that the ratios (18) are successively generated by

$$
\begin{aligned}
k_{n+1}^{(1)} & =\frac{k_{n}^{(3)} \cosh \left[\beta \theta_{n}\right]+k_{n}^{(1)} k_{n}^{(2)} \cosh \left[\beta\left(\theta_{n}-2 J\right)\right]}{k_{n}^{(3)} \cosh \left[\beta\left(\theta_{n}+2 J\right)\right]+k_{n}^{(1)} k_{n}^{(2)} \cosh \left[\beta \theta_{n}\right]} \\
k_{n+1}^{(2)} & =\mathrm{e}^{-2 \beta \theta_{n}} k_{n}^{(1)} k_{n}^{(3)} \frac{\cosh \left[\beta \theta_{n}\right]+k_{n}^{(2)} \cosh \left[\beta\left(\theta_{n}-2 J\right)\right]}{k_{n}^{(3)} \cosh \left[\beta \theta_{n}\right]+k_{n}^{(1)} k_{n}^{(2)} \cosh \left[\beta\left(\theta_{n}-2 J\right)\right]} \\
k_{n+1}^{(3)} & =\frac{\cosh \left[\beta \theta_{n}\right]+k_{n}^{(2)} \cosh \left[\beta\left(\theta_{n}-2 J\right)\right]}{\cosh \left[\beta\left(\theta_{n}+2 J\right)\right]+k_{n}^{(2)} \cosh \left[\beta \theta_{n}\right]} .
\end{aligned}
$$

We note from these iterative equations that if $k_{n}^{(1)}=k_{n}^{(3)}$, then also $k_{n+1}^{(1)}=k_{n+1}^{(3)}$. Furthermore, it follows from identity (13) that $k_{2}^{(1)}=k_{2}^{(3)}$. Thus we are guaranteed that $k_{n}^{(1)}=k_{n}^{(3)}$ for all $n \geqslant 2$, which simplifies our equations considerably:

$k_{n+1}^{(1)}=\frac{\cosh [\beta \tilde{\theta}]+k_{n}^{(2)} \cosh \left[\beta\left(\theta_{n}-2 J\right)\right]}{\cosh \left[\beta\left(\theta_{n}+2 J\right)\right]+k_{n}^{(2)} \cosh [\beta \tilde{\theta}]} \quad k_{n+1}^{(2)}=\mathrm{e}^{-2 \beta \theta_{n}} k_{n}^{(1)} \quad k_{n+1}^{(3)}=k_{n+1}^{(1)}$.

Further substitution of the second of the above equations into the first leads us to a Markovian stochastic process for just a single random variable $k_{n} \equiv k_{n}^{(1)}$ :

$$
k_{n+2}=\psi\left[k_{n} ; \theta_{n+1}, \theta_{n}\right] \quad \psi\left[k^{\prime} ; \theta, \theta^{\prime}\right]=\frac{\cosh [\beta \tilde{\theta}]+\mathrm{e}^{-2 \beta \theta^{\prime}} k^{\prime} \cosh [\beta(\theta-2 J)]}{\cosh [\beta(\theta+2 J)]+\mathrm{e}^{-2 \beta \theta^{\prime}} k^{\prime} \cosh [\beta \tilde{\theta}]} .
$$

In terms of probability densities, the stochastic process (19) can equivalently be written as

$$
P_{i+2}(k)=\frac{1}{4} \sum_{\theta, \theta^{\prime}} \int \mathrm{d} k^{\prime} \delta\left[k-\psi\left[k^{\prime} ; \theta, \theta^{\prime}\right]\right] P_{i}\left(k^{\prime}\right) .
$$


Following in the footsteps of $[2,3]$ we assume the process (20) to be ergodic, and to have a unique stationary state $P_{\infty}(k)=\lim _{i \rightarrow \infty} P_{i}(k)$ (this assumption will turn out to be equivalent to assuming self-averaging of the the free energy per spin, for $N \rightarrow \infty$, with respect to the realization of the random fields). The stationary density $P_{\infty}(k)$ can also be written as the following average over the disorder:

$$
P_{\infty}(k)=\lim _{N \rightarrow \infty} \frac{1}{N} \sum_{i=2}^{N}\left\langle\delta\left[k-\psi\left[k_{i-1} ; \theta_{i}, \theta_{i-1}\right]\right]\right\rangle .
$$

One easily obtains the expression for $P_{\infty}(k)$ for three special (benchmark) cases $\tilde{\theta}=0$ (no external fields), $J=0$ (no spin interactions) and $\theta_{i}=\tilde{\theta} \forall i$ (non-random external fields):

$\tilde{\theta}=0 \quad$ or $\quad J=0: \quad P_{\infty}(k)=\delta[k-1]$

$\theta_{i}=\tilde{\theta} \forall i: \quad P_{\infty}(k)=\delta\left[k+\mathrm{e}^{\beta(2 J+\tilde{\theta})} \sinh (\beta \tilde{\theta})-\mathrm{e}^{\beta \tilde{\theta}} \sqrt{1+\mathrm{e}^{4 \beta J} \sinh ^{2}(\beta \tilde{\theta})}\right]$.

It turns out that both the asymptotic free energy per spin (17) and the asymptotic distribution of local magnetizations (8) can be fully expressed in terms of the stationary distribution $P_{\infty}(k)$ for the random variable $k$, which is what we will demonstrate next. We first invert the relations (18), using $k_{n}^{(3)}=k_{n}^{(1)}$, giving:

$$
\begin{aligned}
& \frac{\mathcal{Z}_{N, \uparrow \downarrow}}{\mathcal{Z}_{N, \uparrow \uparrow}}=\mathrm{e}^{-2 \beta \theta_{N}} k_{N}^{(1)} \quad \frac{\mathcal{Z}_{N, \downarrow \uparrow}}{\mathcal{Z}_{N, \uparrow \uparrow}}=\frac{\cosh \left[\beta\left(\theta_{N}-J\right)\right]}{\cosh \left[\beta\left(\theta_{N}+J\right)\right]} k_{N}^{(2)} \\
& \frac{\mathcal{Z}_{N, \downarrow \downarrow}}{\mathcal{Z}_{N, \uparrow \uparrow}}=\mathrm{e}^{-2 \beta \theta_{N}} \frac{\cosh \left[\beta\left(\theta_{N}-J\right)\right]}{\cosh \left[\beta\left(\theta_{N}+J\right)\right]} k_{N}^{(1)} k_{N}^{(2)} .
\end{aligned}
$$

Using the strict positivity of $k_{n}^{(1)}$ we find that the outcome of the mapping $\psi[k ; \ldots]$ (19) lies always in the interval $\left[k_{\text {low }}, k_{\text {up }}\right]$, where $k_{\text {low }}=k_{\text {up }}^{-1}=\cosh [\beta \tilde{\theta}] / \cosh [\beta(\tilde{\theta}+2 J)]$. Thus the quantities $k_{n}^{(1)}$ and $k_{n}^{(2)}$ are bounded, one consequence of which is that we can write (17) as

$$
\begin{aligned}
f_{\infty}=-\lim _{N \rightarrow \infty} & \frac{1}{\beta N} \log \left\{\mathcal { Z } _ { N , \uparrow \uparrow } \left[1+\mathrm{e}^{-2 \beta \theta_{N}} k_{N}^{(1)}+\frac{\cosh \left[\beta\left(\theta_{N}-J\right)\right]}{\cosh \left[\beta\left(\theta_{N}+J\right)\right]} k_{N}^{(2)}\right.\right. \\
& \left.\left.+\mathrm{e}^{-2 \beta \theta_{N}} \frac{\cosh \left[\beta\left(\theta_{N}-J\right)\right]}{\cosh \left[\beta\left(\theta_{N}+J\right)\right]} k_{N}^{(1)} k_{N}^{(2)}\right]\right\} \\
= & -\lim _{N \rightarrow \infty} \frac{1}{\beta N} \log \mathcal{Z}_{N, \uparrow \uparrow .}
\end{aligned}
$$

This expression, which could also have been derived for any of the three other conditional partition functions, just confirms that the asymptotic free energy is independent of our enforcing of the states of the last two spins in the chain. Next we use (14):

$$
\begin{gathered}
\lim _{N \rightarrow \infty} \frac{1}{\beta N} \log \mathcal{Z}_{N, \uparrow \uparrow}=\lim _{N \rightarrow \infty} \frac{1}{\beta N} \log \left\{M_{11}^{+}\left[\theta_{N}, \theta_{N-1}\right] \mathcal{Z}_{N-1, \uparrow \uparrow}+M_{12}^{+}\left[\theta_{N}, \theta_{N-1}\right] \mathcal{Z}_{N-1, \downarrow \uparrow}\right\} \\
=\lim _{N \rightarrow \infty} \frac{1}{\beta N} \log \left\{\mathcal { Z } _ { N - 1 , \uparrow \uparrow } \left[M_{11}^{+}\left[\theta_{N}, \theta_{N-1}\right]\right.\right. \\
\left.\left.\quad+M_{12}^{+}\left[\theta_{N}, \theta_{N-1}\right] \frac{\cosh \left[\beta\left(\theta_{N-1}-J\right)\right]}{\cosh \left[\beta\left(\theta_{N-1}+J\right)\right]} k_{N-2} \mathrm{e}^{-2 \beta \theta_{N-2}}\right]\right\} .
\end{gathered}
$$

Repetition of this argument, descending fully along the chain, allows us to completely decompose the conditional partition function $\mathcal{Z}_{N, \uparrow \uparrow}$ and arrive at

$$
\begin{aligned}
\lim _{N \rightarrow \infty} \frac{1}{\beta N} \log \mathcal{Z}_{N, \uparrow \uparrow}=\lim _{N \rightarrow \infty} \frac{1}{\beta N} \sum_{i=3}^{N} \log \left\{M_{11}^{+}\left[\theta_{i}, \theta_{i-1}\right]\right. \\
\left.+M_{12}^{+}\left[\theta_{i}, \theta_{i-1}\right] \frac{\cosh \left[\beta\left(\theta_{i-1}-J\right)\right]}{\cosh \left[\beta\left(\theta_{i-1}+J\right)\right]} k_{i-2} \mathrm{e}^{-2 \beta \theta_{i-2}}\right\} .
\end{aligned}
$$


Substitution from (15) of the matrix elements of $M^{+}\left[\theta_{i}, \theta_{i-1}\right]$ and insertion into (24) then leads to the desired result:

$$
f_{\infty}=-\frac{1}{\beta} \log 2 \cosh [\beta \tilde{\theta}]-\lim _{N \rightarrow \infty} \frac{1}{\beta N} \sum_{i=3}^{N} \log \left\{\frac{\cosh \left[\beta\left(\theta_{i-1}+2 J\right)\right]}{\cosh [\beta \tilde{\theta}]}+k_{i-2} \mathrm{e}^{-2 \beta \theta_{i-2}}\right\} .
$$

Since each $k_{i}$ only depends on random fields $\theta_{j}$ with $j<i$, the explicit occurrences of $\left\{\theta_{i-1}\right\}$ and $\left\{\theta_{i-2}\right\}$ are automatically averaged out, independently of the $\left\{k_{i-2}\right\}$, giving

$$
\begin{aligned}
f_{\infty}=-\frac{1}{\beta} \log 2 & \cosh [\beta \tilde{\theta}]-\frac{1}{4 \beta} \int \mathrm{d} k P_{\infty}(k) \log \{F(k, \tilde{\theta}, \tilde{\theta}) F(k,-\tilde{\theta}, \tilde{\theta}) \\
\times & F(k, \tilde{\theta},-\tilde{\theta}) F(k,-\tilde{\theta},-\tilde{\theta})\}
\end{aligned}
$$

with

$$
F\left(k, \theta^{\prime}, \theta\right)=\frac{\cosh \left[\beta\left(\theta^{\prime}+2 J\right)\right]}{\cosh [\beta \tilde{\theta}]}+k \mathrm{e}^{2 \beta \theta} .
$$

This confirms that the solution of the model has indeed been reduced to determining the stationary distribution $P_{\infty}(k)$ of the stochastic map (19), as claimed. In the absence of either the random fields or the interaction couplings we would find (20) evolving into $P_{\infty}(k)=\delta[k-1]$ and the free energy (25) would reduce to $f_{\infty}=-\frac{2}{\beta} \log 2 \cosh [\beta J]$, as it should (cf the limit $\theta \rightarrow 0$ of the short-range/non-random field equations and the limit $J \rightarrow 0$ of the infiniterange/random-field equations in [21]).

\subsection{Statistics of local observables}

Finally, in order to calculate the field distribution (8) and clarify the physical meaning of the function $P_{\infty}(k)$ we now turn to the local magnetizations $\left\langle\sigma_{\ell}\right\rangle$ and the next-time nearestneighbour correlation functions $\left\langle\sigma_{\ell-1} \tanh \left[\beta h_{\ell}(\sigma)\right]\right\rangle$, and show that they can be expressed in terms of the conditional partition functions (12). This is achieved by exploiting the symmetry $\Omega_{i}\left[\sigma_{i-1}, \sigma_{i}, \sigma_{i+1}\right]=\Omega_{i}\left[\sigma_{i+1}, \sigma_{i}, \sigma_{i-1}\right]$, which allows us to write both $\left\langle\sigma_{\ell}\right\rangle$ and $\left\langle\sigma_{\ell-1} \tanh \left[\beta h_{\ell}(\sigma)\right]\right\rangle$ in terms of two sub-chains which start at the left- and right-hand side of the original $N$-spin chain, of length $\ell$ and $N-\ell+1$ respectively, and which connect at site $\ell$. We will take $\ell$ to be an internal site, i.e. $2<\ell<N-1$. Using the notation introduced earlier (10) we obtain for the local magnetization

$$
\begin{aligned}
\left\langle\sigma_{\ell}\right\rangle=\frac{\sum_{\sigma_{1} \ldots \sigma_{N}} \Omega_{1}\left[0, \sigma_{1}, \sigma_{2}\right] \prod_{i=2}^{N-1} \Omega_{i}\left[\sigma_{i-1}, \sigma_{i}, \sigma_{i+1}\right] \Omega_{N}\left[\sigma_{N-1}, \sigma_{N}, 0\right] \sigma_{\ell}}{\mathcal{Z}_{N, \uparrow \uparrow}+\mathcal{Z}_{N, \uparrow \downarrow}+\mathcal{Z}_{N, \downarrow \uparrow}+\mathcal{Z}_{N, \downarrow \downarrow}} \\
=\frac{\mathrm{e}^{-\beta \theta_{\ell}} \sum_{\lambda \tau \in\{\uparrow \downarrow\}} \gamma_{\lambda \tau}^{(\ell)} \mathcal{Z}_{\ell, \lambda \uparrow} \mathcal{Z}_{N-\ell+1, \tau \uparrow}-\mathrm{e}^{\beta \theta_{\ell}} \sum_{\lambda \tau \in\{\uparrow \downarrow\}} \gamma_{\lambda \tau}^{(\ell)} \mathcal{Z}_{\ell, \lambda \downarrow} \mathcal{Z}_{N-\ell+1, \tau \downarrow}}{\mathrm{e}^{-\beta \theta_{\ell}} \sum_{\lambda \tau \in\{\uparrow \downarrow\}} \gamma_{\lambda \tau}^{(\ell)} \mathcal{Z}_{\ell, \lambda \uparrow} \mathcal{Z}_{N-\ell+1, \tau \uparrow}+\mathrm{e}^{\beta \theta_{\ell}} \sum_{\lambda \tau \in\{\uparrow \downarrow\}} \gamma_{\lambda \tau}^{(\ell)} \mathcal{Z}_{\ell, \lambda \downarrow} \mathcal{Z}_{N-\ell+1, \tau \downarrow}}
\end{aligned}
$$

and for the local next-time nearest-neighbour correlations

$$
\begin{array}{r}
\left\langle\sigma_{\ell-1} \tanh \left[\beta h_{\ell}(\sigma)\right]\right\rangle \\
=\frac{\mathrm{e}^{-\beta \theta_{\ell}} \sum_{\lambda \tau \in\{\uparrow \downarrow\}} \chi_{\lambda \tau}^{(\ell)} \mathcal{Z}_{\ell, \lambda \uparrow} \mathcal{Z}_{N-\ell+1, \tau \uparrow}+\mathrm{e}^{\beta \theta_{\ell}} \sum_{\lambda \tau \in\{\uparrow \downarrow\}} \chi_{\lambda \tau}^{(\ell)} \mathcal{Z}_{\ell, \lambda \downarrow} \mathcal{Z}_{N-\ell+1, \tau \downarrow}}{\mathrm{e}^{-\beta \theta_{\ell}} \sum_{\lambda \tau \in\{\uparrow \downarrow\}} \chi_{\lambda \tau}^{(\ell)} \mathcal{Z}_{\ell, \lambda \uparrow} \mathcal{Z}_{N-\ell+1, \tau \uparrow}+\mathrm{e}^{\beta \theta_{\ell}} \sum_{\lambda \tau \in\{\uparrow \downarrow\}} \chi_{\lambda \tau}^{(\ell)} \mathcal{Z}_{\ell, \lambda \downarrow} \mathcal{Z}_{N-\ell+1, \tau \downarrow}}
\end{array}
$$

in which

$$
\gamma_{\lambda \tau}^{(\ell)}=\frac{\cosh \left[\beta\left(\theta_{\ell}+J(\lambda+\tau)\right)\right]}{\cosh \left[\beta\left(\theta_{\ell}+J \lambda\right)\right] \cosh \left[\beta\left(\theta_{\ell}+J \tau\right)\right]} \quad \chi_{\lambda \tau}^{(\ell)}=\frac{\lambda \sinh \left[\beta\left(\theta_{\ell}+J(\lambda+\tau)\right)\right]}{\cosh \left[\beta\left(\theta_{\ell}+J \lambda\right)\right] \cosh \left[\beta\left(\theta_{\ell}+J \tau\right)\right]} .
$$

Both the sub-chain generated from the left, with $\ell$ spins, and the sub-chain generated from the right, with $N-\ell+1$ spins, will find at their endpoint sites their common random field $\theta_{\ell}$. 
Apart from this particular site, however, their remaining random fields are fully independent. We divide the numerator and denominator by $\mathcal{Z}_{\ell, \uparrow \uparrow} \mathcal{Z}_{N-\ell+1, \uparrow \uparrow}$, and use (22), (23) to eliminate the conditional partition functions in favour of the two statistically independent sets of ratios $\left\{k_{\ell}^{(n)}\right\}$ and $\left\{k_{N-\ell+1}^{(n)}\right\}$. After some serious yet unpleasant book-keeping we then end up with

$$
\begin{aligned}
& \left\langle\sigma_{\ell}\right\rangle=\frac{\mathrm{e}^{2 \beta \theta_{\ell}}-k_{N-\ell+1} k_{\ell}}{\mathrm{e}^{2 \beta \theta_{\ell}}+k_{N-\ell+1} k_{\ell}}=\frac{\mathrm{e}^{2 \beta \theta_{\ell}}-\psi\left[k_{N-\ell+3} ; \theta_{N-\ell+2}, \theta_{N-\ell+3}\right] \psi\left[k_{\ell-2} ; \theta_{\ell-1}, \theta_{\ell-2}\right]}{\mathrm{e}^{2 \beta \theta_{\ell}}+\psi\left[k_{N-\ell+3} ; \theta_{N-\ell+2}, \theta_{N-\ell+3}\right] \psi\left[k_{\ell-2} ; \theta_{\ell-1}, \theta_{\ell-2}\right]} \\
& \left\langle\sigma_{\ell-1} \tanh \left[\beta h_{\ell}(\sigma)\right]\right\rangle \\
& \quad=\frac{\sinh \left[\beta\left(2 J+\theta_{\ell}\right)\right]+\sinh \left[\beta \theta_{\ell}\right]\left(k_{N-\ell+1}^{(2)}-k_{\ell}^{(2)}\right)+\sinh \left[\beta\left(2 J-\theta_{\ell}\right)\right] k_{N-\ell+1}^{(2)} k_{\ell}^{(2)}}{\cosh \left[\beta\left(2 J+\theta_{\ell}\right)\right]+\cosh \left[\beta \theta_{\ell}\right]\left(k_{N-\ell+1}^{(2)}+k_{\ell}^{(2)}\right)+\cosh \left[\beta\left(2 J-\theta_{\ell}\right)\right] k_{N-\ell+1}^{(2)} k_{\ell}^{(2)}}
\end{aligned}
$$

where $k_{\ell}^{(2)}=k_{\ell-1} \mathrm{e}^{-2 \beta \theta_{\ell-1}}$ and $k_{N-\ell+1}^{(2)}=k_{N-\ell+2} \mathrm{e}^{-2 \beta \theta_{N-\ell+2}}$ and with the function $\psi[\ldots]$ as defined in (19).

We now carry out a benchmark test of these results by comparing them with two simple solvable cases, namely the synchronous infinite-range/random-field model and the synchronous short-range/non-random field model as studied in [21]. Firstly, for $J=0$ the random variable $k$ evolves towards the fixed point $k=1$ and the above expressions for the local observables indeed reproduce the correct expressions $\left\langle\sigma_{\ell}\right\rangle=\tanh \left[\beta \theta_{\ell}\right]$ and $\left\langle\sigma_{\ell-1} \tanh \left[\beta h_{\ell}(\sigma)\right]\right\rangle=\tanh \left[\beta \theta_{\ell-1}\right] \tanh \left[\beta \theta_{\ell}\right]$ (cf with the limit $J \rightarrow 0$ of the infiniterange/random-field equations in [21]). Secondly, in the absence of external fields we have $k=1$, which again reproduces the correct results $\left\langle\sigma_{\ell}\right\rangle=0$ and $\left\langle\sigma_{\ell-1} \tanh \left[\beta h_{\ell}(\sigma)\right]\right\rangle=0$ (cf with the limit $\tilde{\theta} \rightarrow 0$ of the short-range/non-random field equations in [21]). Finally for uniform external fields, i.e. for $\theta_{i}=\tilde{\theta} \forall i$ or $\theta_{i}=-\tilde{\theta} \forall i$ we find $P_{\infty}(k)$ evolving towards the delta peak given by (21) which, combined with the above expresions for the local observables, can again be shown to reproduce observables (7) as found via the transfer-matrix analysis of the short-range/non-random field model in [21].

\subsection{Link between synchronous and sequential dynamics}

In the two special cases of synchronous dynamics models (infinite-range interactions and random fields, short-range interactions and non-random fields) as studied in [21] we found a simple relation between the transfer matrices $\left(\boldsymbol{T}_{\mathrm{syn}}=\boldsymbol{T}_{\text {seg }}^{2}\right)$ and the free energies per spin $\left(f_{\text {syn }}=2 f_{\text {seq }}\right)$ corresponding to parallel (synchronous) and sequential dynamics. We now inspect the relation between the equilibria following synchronous versus sequential dynamics in short-range random-field models. We have seen that in the thermodynamic limit all intensive quantities ultimately follow from the stationary distribution $P_{\infty}(k)$ of the random variable $k$. We note that the stochastic process (19) underlying this random variable overlooks nearest neighbours and thus distinguishes between even and odd sites:

$$
\begin{array}{lc}
\text { synchronous: } & k_{n+2}=\psi_{\mathrm{syn}}\left[k_{n} ; \theta_{n+1}, \theta_{n}\right] \\
\psi_{\mathrm{syn}}\left[k^{\prime} ; \theta, \theta^{\prime}\right]=\frac{\cosh [\beta \tilde{\theta}]+\mathrm{e}^{-2 \beta \theta^{\prime}} k^{\prime} \cosh [\beta(\theta-2 J)]}{\cosh [\beta(\theta+2 J)]+\mathrm{e}^{-2 \beta \theta^{\prime}} k^{\prime} \cosh [\beta \tilde{\theta}]} .
\end{array}
$$

This appears to be in sharp contrast with sequential Glauber-type random-field models, where the one-dimensional RFIM analysis [2,3] leads to the following stochastic process:

sequential: $\quad k_{n+1}=\psi_{\text {seq }}\left[k_{n} ; \theta_{n}\right] \quad \psi_{\text {seq }}\left[k^{\prime} ; \theta^{\prime}\right]=\frac{\mathrm{e}^{-\beta J}+k^{\prime} \mathrm{e}^{\beta J} \mathrm{e}^{-2 \beta \theta^{\prime}}}{\mathrm{e}^{\beta J}+k^{\prime} \mathrm{e}^{-\beta J} \mathrm{e}^{-2 \beta \theta^{\prime}}}$. 
It turns out that the link between the two processes is made via the following identity, which can be verified by explicit substitution:

$$
\psi_{\text {seq }}\left[\psi_{\text {seq }}\left[k^{\prime} ; \theta^{\prime}\right] ; \theta\right]=\psi_{\text {syn }}\left[k^{\prime} ; \theta, \theta^{\prime}\right] .
$$

This relation, in turn, immediately allows us to derive the identity $P_{\infty}^{\mathrm{seq}}(k)=P_{\infty}^{\mathrm{syn}}(k)$. To see this we express the probability distribution $P_{i+2}^{\text {seq }}(k)$ in terms of $P_{i}^{\text {seq }}(k)$ :

$$
\begin{aligned}
P_{i+2}^{\mathrm{seq}}(k) & =\frac{1}{4} \sum_{\theta^{\prime}, \theta^{\prime \prime}} \int \mathrm{d} k^{\prime} \mathrm{d} k^{\prime \prime} \delta\left[k^{\prime}-\psi_{\mathrm{seq}}\left[k^{\prime \prime} ; \theta^{\prime \prime}\right]\right] \delta\left[k-\psi_{\mathrm{seq}}\left[k^{\prime} ; \theta^{\prime}\right]\right] P_{i}^{\mathrm{seq}}\left(k^{\prime \prime}\right) \\
& =\frac{1}{4} \sum_{\theta^{\prime}, \theta^{\prime \prime}} \int \mathrm{d} k^{\prime \prime} \delta\left[k-\psi_{\mathrm{seq}}\left[\psi_{\mathrm{seq}}\left[k^{\prime \prime} ; \theta^{\prime \prime}\right] ; \theta^{\prime}\right]\right] P_{i}^{\mathrm{seq}}\left(k^{\prime \prime}\right) .
\end{aligned}
$$

The transition probabilities in the last equation can be seen to have become exactly those defined in (20) for synchronous dynamics. Thus the two probability destributions $P_{i}^{\text {seq }}(k)$ and $P_{i}^{\text {syn }}(k)$ basically describe the evolution of the same random variable, giving (upon assuming uniqueness of the stationary state) $P_{\infty}^{\mathrm{seq}}(k)=P_{\infty}^{\mathrm{syn}}(k)$.

Let us finally turn to integrated densities $\hat{P}_{i}(k)=\int_{0}^{k} \mathrm{~d} z P_{i}(z)$, in order to establish contact with the solution of [3], and to appreciate in this alternative representation the equivalence of sequential and synchronous dynamics. For the synchronous process (20) we now have

$$
\begin{aligned}
\hat{P}_{i+2}^{\text {syn }}(k) & =\frac{1}{4} \sum_{\theta, \theta^{\prime}} \int_{0}^{\infty} \mathrm{d} k \int_{0}^{k} \mathrm{~d} z \delta\left[z-\psi_{\text {syn }}\left[k ; \theta, \theta^{\prime}\right]\right] P_{i}^{\text {syn }}(k) \\
& =\frac{1}{4} \sum_{\theta, \theta^{\prime}} \int_{0}^{k} \mathrm{~d} z\left|\frac{\partial g_{\text {syn }}\left(z, \theta, \theta^{\prime}\right)}{\partial z}\right| P_{i}^{\text {syn }}\left(g_{\text {syn }}\left(z, \theta, \theta^{\prime}\right)\right)
\end{aligned}
$$

where

$$
g_{\text {syn }}\left(z, \theta, \theta^{\prime}\right)=\mathrm{e}^{2 \beta \theta^{\prime}} \frac{z \cosh [\beta(\theta+2 J)]-\cosh [\beta \tilde{\theta}]}{\cosh [\beta(\theta-2 J)]-z \cosh [\beta \tilde{\theta}]} .
$$

This allows us to now bring the integrated densities in the closed recurrence form:

$$
\begin{aligned}
& \hat{P}_{i+2}^{\text {syn }}(k)=\mathcal{K}_{\text {syn }}\left[\hat{P}_{i}^{\text {syn }}(k)\right] \\
& \mathcal{K}_{\text {syn }}[L(k)]= \frac{1}{4}\left\{L\left(g_{\text {syn }}(k, \tilde{\theta}, \tilde{\theta})\right)+L\left(g_{\text {syn }}(k, \tilde{\theta},-\tilde{\theta})\right)\right. \\
&\left.+L\left(g_{\text {syn }}(k,-\tilde{\theta}, \tilde{\theta})\right)+L\left(g_{\text {syn }}(k,-\tilde{\theta},-\tilde{\theta})\right)\right\} .
\end{aligned}
$$

We note that the corresponding sequential random-field expression found in $[2,3]$ is

$$
\begin{aligned}
& \hat{P}_{i+1}^{\text {seq }}(k)=\mathcal{K}_{\text {seq }}\left[\hat{P}_{i}^{\text {seq }}(k)\right] \\
& \mathcal{K}_{\text {seq }}[L(k)]=\frac{1}{2}\left\{L\left(g_{\text {seq }}(k, \tilde{\theta})\right)+L\left(g_{\text {seq }}(k,-\tilde{\theta})\right)\right\} \\
& g_{\text {seq }}(k, \theta)=\mathrm{e}^{2 \beta \theta} \frac{k \mathrm{e}^{\beta J}-\mathrm{e}^{-\beta J}}{\mathrm{e}^{\beta J}-k \mathrm{e}^{-\beta J}}
\end{aligned}
$$

and it can be easily verified that $g_{\text {syn }}\left(k, \theta, \theta^{\prime}\right)=g_{\text {seq }}\left(g_{\text {seq }}(k, \theta), \theta^{\prime}\right)$, which implies

$$
\mathcal{K}_{\text {seq }}\left[\mathcal{K}_{\text {seq }}\left[\hat{P}_{i}(k)\right]\right]=\mathcal{K}_{\text {syn }}\left[\hat{P}_{i}(k)\right]
$$

\section{Physical properties of the model}

\subsection{The devil's staircase and the free energy}

It has been found in [3] that for $\mathrm{e}^{2 \beta J}<1+2 \cosh [2 \beta \tilde{\theta}]$ the sequential dynamics integrated distribution function $\hat{P}_{\infty}^{\text {seq }}(k)$ acquires the form of a devil's staircase. Since our analysis has shown, upon suitable definition of the ratio $k$ for synchronous dynamics, that the stationary 
distributions $P_{\infty}(k)$ of synchronous and sequential dynamics are identical, we will derive our results for the synchronous RFIM, wherever possible, from the (less complicated) sequential dynamics relation (31). The construction of the devil's staircase solution can be found in [3] (see also the much earlier paper [22]). It is based on the successive application of the operator $\mathcal{K}_{\text {seq }}$ on $\hat{P}_{i-m}(k)$ with $m=0,1, \ldots, \infty$, and the derivation of upper and lower bounds for the intervals over which $\hat{P}_{i-m}(k)$ is constant. At stage $m$ of this procedure one obtains $\ell=1, \ldots, 2^{m}$ special intervals, which will be denoted by $\Delta_{m}^{\ell}$, such that $\forall k \in \Delta_{m}^{\ell}$ one has $\hat{P}_{i+1}(k)=(2 \ell-1) / 2^{m+1}$. The largest of these intervals is generated at $m=0: \forall k \in \Delta_{0}^{1}, \hat{P}_{i+1}(k)=\frac{1}{2}$. The lower and upper endpoints of an interval $\Delta_{m}^{\ell}$, denoted by $\Delta_{m}^{\ell_{L}}$ and $\Delta_{m}^{\ell_{U}}$, are generated by the recurrent process:

$$
\begin{array}{ll}
\Delta_{m+1}^{(2 \ell-1)_{L}}=G\left(\Delta_{m}^{\ell_{L}}, \tilde{\theta}\right) & \Delta_{m+1}^{(2 \ell-1)_{U}}=G\left(\Delta_{m}^{\ell_{U}}, \tilde{\theta}\right) \\
\Delta_{m+1}^{(2 \ell)_{L}}=G\left(\Delta_{m}^{\ell_{L}},-\tilde{\theta}\right) & \Delta_{m+1}^{(2 \ell)_{U}}=G\left(\Delta_{m}^{\ell_{U}},-\tilde{\theta}\right)
\end{array}
$$

where $G^{\text {inv }}(k, \pm \tilde{\theta})=g_{\text {seq }}(k, \pm \tilde{\theta})$, and with initial values

$$
\begin{aligned}
\Delta_{0}^{1_{L}} & =G\left(\Delta_{U}, \tilde{\theta}\right) & \Delta_{L} & =g_{\text {seq }}\left(\Delta_{L}, \tilde{\theta}\right) \\
\Delta_{0}^{1_{U}} & =G\left(\Delta_{L},-\tilde{\theta}\right) & \Delta_{U} & =g_{\text {seq }}\left(\Delta_{U},-\tilde{\theta}\right)
\end{aligned}
$$

where $\Delta_{L}$ and $\Delta_{U}$ represent the points with the property: $\forall k<\Delta_{L}, \hat{P}_{i+1}(k)=0$ and $\forall k>\Delta_{U}$, $\hat{P}_{i+1}(k)=1$. For small $\tilde{\theta} / J$ the support of $P_{\infty}(k)$, denoted by $\Delta=\cup_{m, \ell_{L}, \ell_{U}}\left\{\Delta_{m}^{\ell_{L}}, \Delta_{m}^{\ell_{U}}\right\}$ forms a fully connected set, namely: $\Delta=\left[\Delta_{L}, \Delta_{U}\right]$. As the ratio $\tilde{\theta} / J$ increases, and the strength of the random fields starts to dominate the spin couplings, $\Delta$ becomes disconnected. From the point onwards where the difference $\Delta_{0}^{1_{U}}-\Delta_{0}^{1_{L}}$ first becomes non-negative (at $\left.\mathrm{e}^{2 \beta J}=1+2 \cosh [2 \beta \tilde{\theta}]\right) \hat{P}_{\infty}(k)$ will take the form of a devil's staircase, and $\Delta$ itself will turn into the Cantor set with fractal dimension $D_{F}<1$, see also [4,9]. Figure 1 $(a)$ shows how the set $\Delta$ changes as the ratio $\tilde{\theta} / J$ is varied. The vertical axis corresponds to the normalized quantity $\tilde{k}=(k-1) /(k+1) \in[-1,1]$. This graph has been drawn for 10000 iterations of the synchronous stochastic map (19). White regions correspond to ratios $k$ for which $P(k)<0.0001$, i.e. non-attainable values of $\psi_{\text {syn }}\left[k^{\prime} ; \theta, \theta^{\prime}\right]$. These are already seen to be present for $\Delta_{0}^{1_{U}}-\Delta_{0}^{1_{L}}<0$, and correspond to plateaus of the integrated density $\hat{P}(k)$ (see figure $2(b)$ ). For $\Delta_{0}^{1_{U}}-\Delta_{0}^{1_{L}} \geqslant 0$ the graph shows distinct pairs of branches (for positive and negative $\tilde{k}$ ) which correspond to the endpoints of the interval-bands $\Delta_{m}^{\ell_{L}}(\tilde{\theta} / J)$ and $\Delta_{m}^{\ell_{U}}(\tilde{\theta} / J)$ for all $m, \ell$. For large $\tilde{\theta} / J$ the length of the interval $\Delta_{0}^{1}$ approaches $\Delta_{U}-\Delta_{L}$ while the length of all other interval-bands $\Delta_{m}^{\ell}$ tends to zero. $\hat{P}(k)$ then approaches a simple step function, with $\hat{P}(k)=\frac{1}{2}$ for all $k \in\left[\Delta_{L}, \Delta_{U}\right]$. Figure 1 also suggests that $P_{\infty}(k)$ is symmetric when plotted against $\tilde{k}$. This property can be derived analytically from the identity $g_{\text {seq }}(k, \tilde{\theta})=1 / g_{\text {seq }}(1 / k,-\tilde{\theta})$ (by using it to verify via induction with respect to $n$ that $\left.\hat{P}_{n}(k)+\hat{P}_{n}(1 / k)=1\right)$, in combination with the assumed ergodicity of the stochastic process for $k$ and uniqueness of $P_{\infty}(k)$.

Figures 3 and 4 show the supports of the probability densities for the local observables $\left\langle\sigma_{\ell}\right\rangle$ and $\left\langle\sigma_{\ell} \tanh \left[\beta \sigma_{\ell-1}(\sigma)\right]\right\rangle$, plotted against $\tilde{\theta} / J$. For the local magnetization this support eventually becomes a Cantor set, for large $\tilde{\theta} / J$, although not at the same transition line at which the support of $P(k)$ does so. This has also been noted for the sequential RFIM in [4], and is due to the fact that the expression (26) for $\left\langle\sigma_{\ell}\right\rangle$ involves the product of $t w o$ stochastic variables, namely $k_{\ell}$ and $k_{N-\ell+1}$. In figure 4 , dealing with next-time nearest-neighbour correlations, we see that, due to the choice of ferromagnetic interactions (here $J=2.1$ ), a spin at site $\ell$ tends to align with its neighbours at a previous time step, leading to a positive bias for these correlations. For sufficiently small random field strengths virually all $\left\langle\sigma_{\ell} \tanh \left[\beta h_{\ell-1}(\sigma)\right]\right\rangle$ 

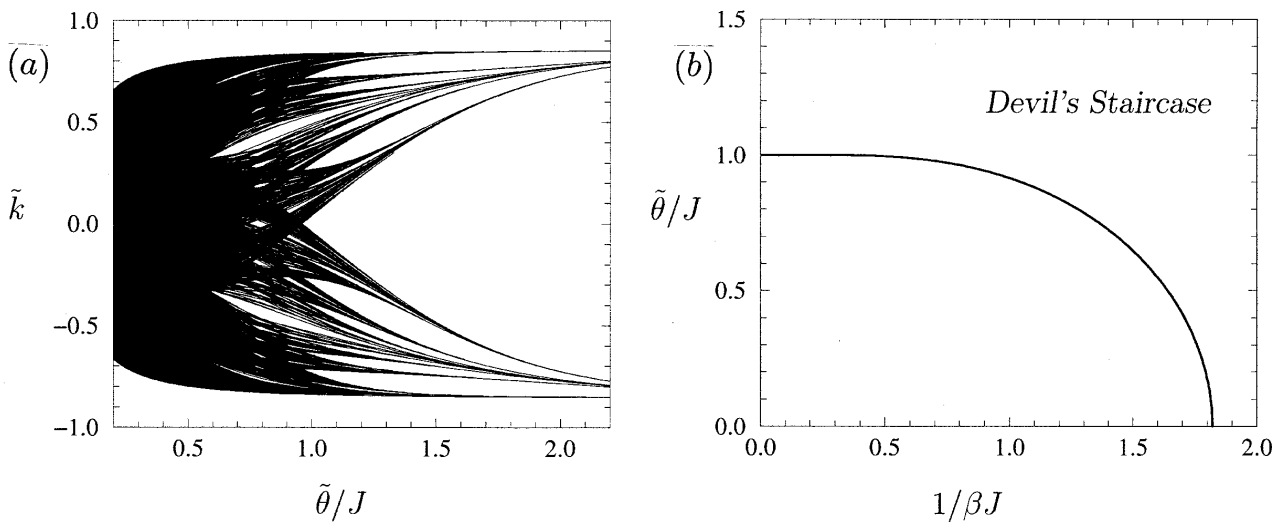

Figure 1. (a) Graphical representation of the set $\Delta$ of possible values of the stochastic variable $k$, as a function of the ratio $\tilde{\theta} / J$, for $\beta=0.6$. It is constructed by performing 10000 iterations of the stochastic map (19). The vertical axis corresponds to the (normalized) quantity $\tilde{k}=(k-1) /(k+1)$. White regions correspond to values of $k$ for which $P(k)<0.0001$, also showing up as plateaus in figure 2. The set $\Delta$ becomes a Cantor set, and thus $\hat{P}(k)$ a devil's staircase, for $\Delta_{0}^{1_{U}}-\Delta_{0}^{1_{L}} \geqslant 0$ (here: $\tilde{\theta} / J \approx 0.96$ ). (b) Phase diagram showing the transition line $\Delta_{0}^{1_{U}}-\Delta_{0}^{1_{L}}=0$ where $\hat{P}(k)$ takes the form of devil's staircase. Note that the fractal dimension of the support of $P(k)$ is already less than one before the transition to a devil's staircase occurs.
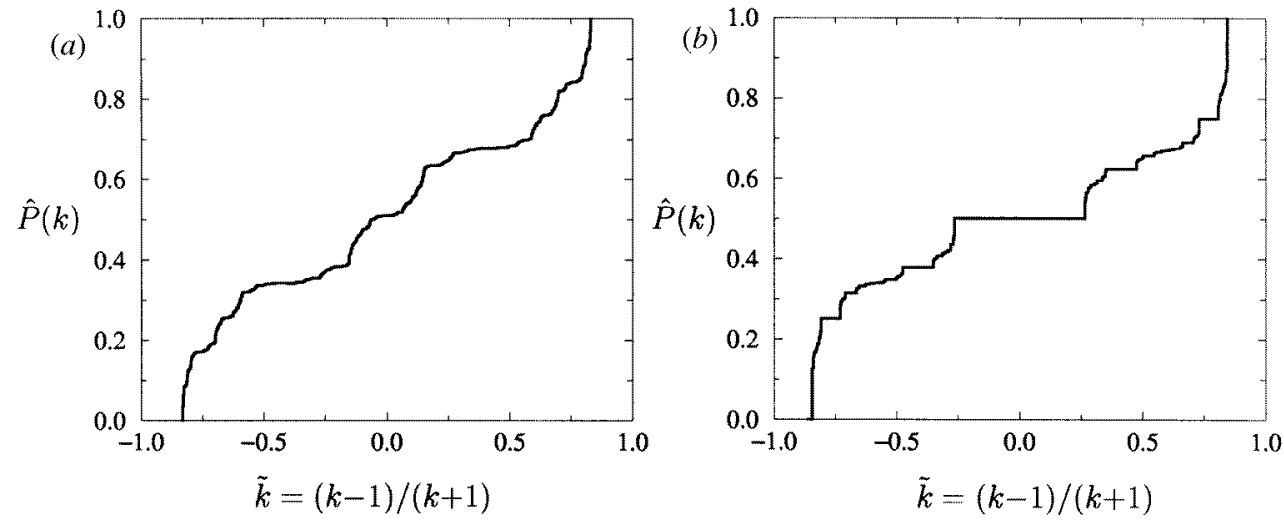

Figure 2. The integrated probability density $\hat{P}(k)$ as a function of the (normalized) variable $\tilde{k}=(k-1) /(k+1)$, for $\beta=0.6$. (a) Parameters such that $\Delta_{0}^{1_{U}}-\Delta_{0}^{1_{L}}<0$ (here: $\tilde{\theta} / J=0.8$ ). Here $\hat{P}(k)$ is highly non-trivial, although not yet a devil's staircase. One can distinguish pairs of plateaus (for positive and negative $\tilde{k}$ ) for which $\hat{P}(k)$ is constant, which correspond to the white (vertical) segments in figure 1 at the cross-section $\tilde{\theta} / J=0.8$. (b) Parameters such that $\Delta_{0}^{1_{U}}-\Delta_{0}^{1_{L}} \geqslant 0$ (here: $\tilde{\theta} / J=1.23)$. Here $\hat{P}(k)$ has become a complete devil's staircase.

will be positive. For large $\tilde{\theta} / J$, on the other hand, the random fields will dominate, and the $\left\langle\sigma_{\ell} \tanh \left[\beta h_{\ell-1}(\sigma)\right]\right\rangle$ will be equally likely to be positive or negative. Once again the picture of discreteness of the set of allowed local observables emerges for large $\tilde{\theta} / J$. For $\tilde{\theta}=0$, the spin interactions control all spin correlations, and the support of the probability density for the $\left\langle\sigma_{\ell} \tanh \left[\beta h_{\ell-1}(\sigma)\right]\right\rangle$ is just a single point.

Let us finally return to the free energy. Since we know that the integrated density $\hat{P}_{\infty}^{\text {syn }}(k)$ takes the form of a devil's staircase, the corresponding distribution $P_{\infty}^{\text {syn }}(k)$ will be a collection 

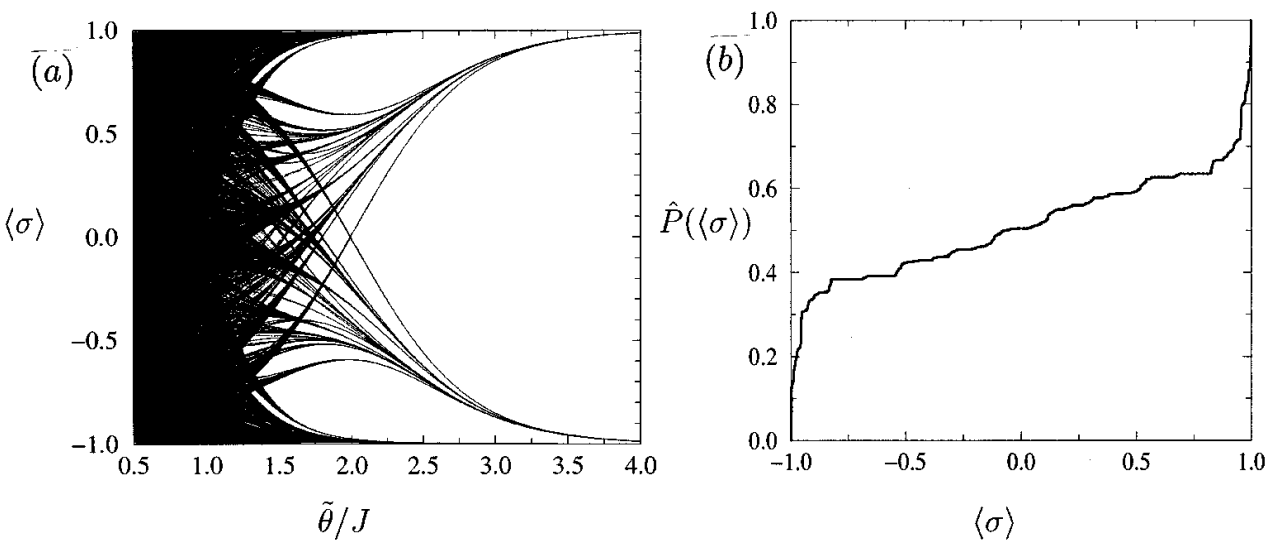

$\langle\sigma\rangle$

Figure 3. (a) Graphical representation of the set of possible values of any local magnetization $\langle\sigma\rangle$ as a function of the ratio $\tilde{\theta} / J$, for $\beta=0.6$ (constructed from expression (26), for chain length $N=10000$ ). For sufficiently small $\tilde{\theta} / J$ one observes a fully connected set, whereas for larger $\tilde{\theta} / J$ it turns into the Cantor set. (b) The integrated probability density for local magnetizations, for $\tilde{\theta}=1.5$; here one already observes plateaus.
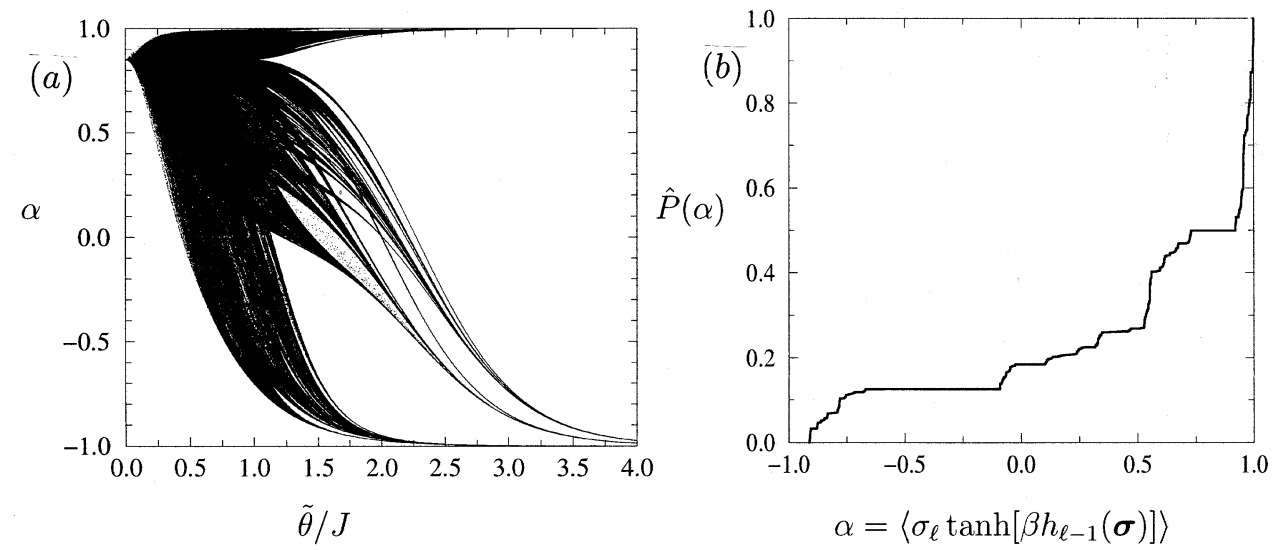

Figure 4. (a) Graphical representation of the set of possible values of any local next-time nearestneighbour correlation $\alpha=\left\langle\sigma_{\ell} \tanh \left[\beta h_{\ell-1}(\sigma)\right]\right\rangle$ as a function of the ratio $\tilde{\theta} / J$, for $\beta=0.6$ (constructed from expression (27), for chain length $N=10000$ ). The graph's bias towards positive values of $\alpha$ is caused by our choice of ferromagnetic interactions: co-alignment with neighbouring sites is preferred, leading to positive correlations. Increasing the ratio $\tilde{\theta} / J$ allows the random fields to dominate spin couplings, and produces highly non-trivial effects on the support of $\alpha$. (b) The integrated probability density of the local next-time nearest-neighbour correlations, for $\tilde{\theta} / J=1.5$.

of delta functions peaked at the endpoints of the intervals $\Delta_{m}^{\ell}$. This allows us to perform the integral over $P_{\infty}(k)$ in (25), and express the free energy formally as

$f_{\infty}=-\frac{1}{\beta} \log 2 \cosh [\beta \tilde{\theta}]+\frac{1}{4 \beta} \Phi\left[\Delta_{U}\right]-\frac{1}{4 \beta} \sum_{m=0}^{\infty} \sum_{\ell=1}^{2^{m}}\left(\frac{2 \ell-1}{2^{m+1}}\right)\left\{\Phi\left[\Delta_{m}^{\ell_{U}}\right]-\Phi\left[\Delta_{m}^{\ell_{L}}\right]\right\}$

where

$$
\Phi(k)=\log \{F(k, \tilde{\theta}, \tilde{\theta}) F(k,-\tilde{\theta}, \tilde{\theta}) F(k, \tilde{\theta},-\tilde{\theta}) F(k,-\tilde{\theta},-\tilde{\theta})\}
$$




$$
F\left(k, \theta^{\prime}, \theta\right)=\frac{\cosh \left[\beta\left(\theta^{\prime}+2 J\right)\right]}{\cosh [\beta \tilde{\theta}]}+k \mathrm{e}^{2 \beta \theta} .
$$

\subsection{Entropy and ground state degeneracy}

Due to the $\beta$-dependence of the pseudo-Hamiltonian (4) which characterizes the equilibrium state of the present model (with synchronous dynamics) we can in principle no longer be sure beforehand of the validity of thermodynamic relations, especially when they involve derivatives with respect to temperature. In this section we will first define and then investigate properties of the entropy, with particular emphasis on its behaviour near the system's ground state. As in ordinary Boltzmann-type situations, one finds that the entropy can be defined via the counting of states (which is, by the way, equivalent to Shannon's information-theoretic definition):

$$
S=-\sum_{\sigma} p_{\infty}(\sigma) \log p_{\infty}(\sigma)
$$

From (33) one easily verifies the validity of $F=U-T S$ upon inserting the equilibrium distribution $p_{\infty}(\sigma)=\mathcal{Z}^{-1} \mathrm{e}^{-\beta \mathcal{H}_{\beta}(\sigma)}$, with Peretto's pseudo-Hamiltonian (4), together with the conventional definitions $\mathcal{Z}=\sum_{\sigma} \mathrm{e}^{-\beta \mathcal{H}_{\beta}(\sigma)}, F=-\frac{1}{\beta} \log \mathcal{Z}_{N}$ and $U=\left\langle\mathcal{H}_{\beta}(\boldsymbol{\sigma})\right\rangle$. However, these self-consistent definitions now imply the identity

$$
S=-\frac{\partial F}{\partial T}+\left\langle\frac{\partial}{\partial T} H_{\beta}(\sigma)\right\rangle .
$$

Working out expression (34) using (4) gives us for the asymptotic entropy per spin $s=$ $\lim _{N \rightarrow \infty} S / N$ :

$$
s=-\frac{\partial f_{\infty}}{\partial T}-\lim _{N \rightarrow \infty} \frac{1}{N} \sum_{i=1}^{N}\left\langle\log 2 \cosh \left[\beta h_{i}(\sigma)\right]-\beta h_{i}(\sigma) \tanh \left[\beta h_{i}(\sigma)\right]\right\rangle .
$$

For $T \rightarrow 0$ the last term is an average over $N$ sites of terms of the form $\lim _{x \rightarrow \infty}|x|[1-$ $\tanh (|x|)]$, each of which goes to zero. Thus, in contrast to the finite-temperature case, the familiar Gibbsian expression for the ground state (zero-temperature) entropy per spin is not affected by the temperature dependence of the Hamiltonian (4):

$$
\lim _{T \rightarrow 0} s=-\lim _{T \rightarrow 0} \frac{\partial f_{\infty}}{\partial T} .
$$

Numerical differentiation of the free energy per spin (25) for increasingly low temperatures shows that the entropy becomes non-zero for $\tilde{\theta} / J \leqslant 2$ while for $\tilde{\theta} / J<2$ an infinite series of transitions appear at

$$
\frac{\tilde{\theta}}{J}=\frac{2}{r} \quad \text { with } \quad r=1,2, \ldots, \infty
$$

where $s$ is relatively large. This type of behaviour has also been noted for Glauber-type random-field systems [5], and is generally interpreted as the fingerprint of a high degree of frustration. As the temperature is gradually decreased towards $T=0$ we find that the peaks in $-\partial f_{\infty} / \partial T$ (as functions of $\tilde{\theta} / J$ ) are smooth local maxima, which become increasingly sharper as $T \rightarrow 0$ (figure 5). Although numerical constraints prevent us from evaluating $\partial f_{\infty} / \partial T$ exactly at $T=0$, the emerging pattern is both transparent and convincing; at the ground state the entropy per spin will depend on $\tilde{\theta} / J$ as an infinite series of delta-type spikes, separated by a monotonic sequence of step functions, as in [5]. 


$$
T=0.01
$$

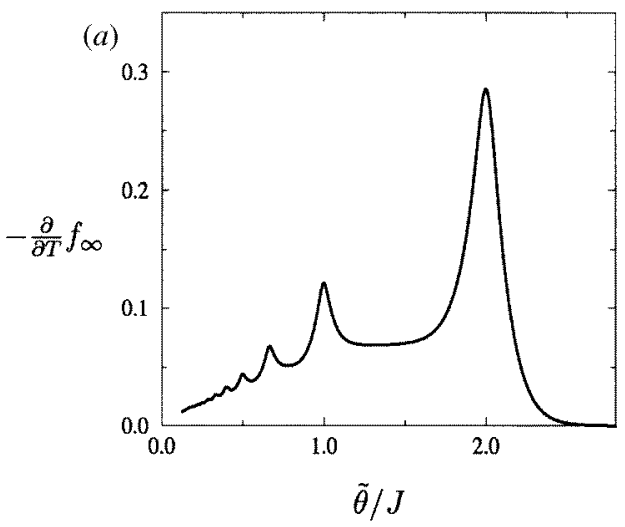

$T=0.003$

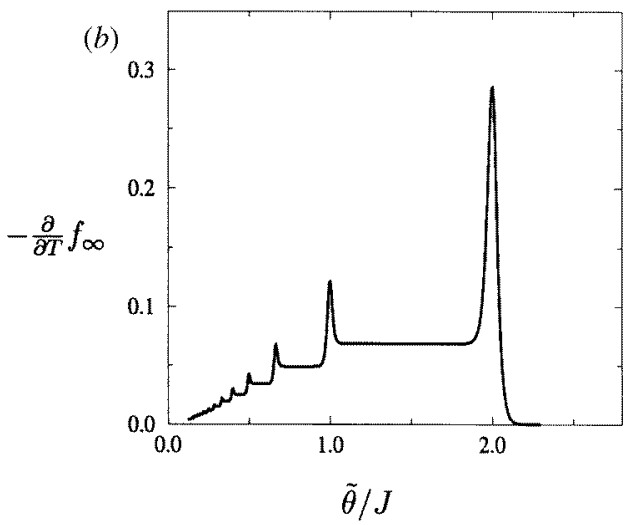

Figure 5. Close to the ground state the function $-\partial f_{\infty} / \partial T$ (which reduces to the entropy per spin $s$ precisely at $T=0$ ) is seen to become non-zero for $\tilde{\theta} / J \leqslant 2$, and to develop an infinite series of smooth local maxima, located at the special ratios $\tilde{\theta} / J=2 / r$ for $r=1,2, \ldots, \infty$. As the temperature is reduced to zero, these maxima sharpen consistently, becoming infinitely sharp peaks at $T=0$.

\section{Discussion}

In this paper we have solved the one-dimensional random field Ising model with synchronous dynamics, using the equilibrium distribution characterized by Peretto's [15] (pseudo-) Hamiltonian and an adaptation of the techniques originally developed for the sequential dynamics RFIM (see, e.g., [2,3]). These techniques are based on deriving autonomous (but stochastic) recurrent relations for conditioned partition functions, expressing those of an $N+1$ spin system (with $N+1$ random fields) in terms of those of an $N$ spin one (with $N$ random fields).

In contrast to the sequential RFIM, we show that for the synchronous dynamics RFIM one needs to condition on the states of the last two spins in the chain, rather than just the last one, in order to arrive at autonomous recurrent stochastic relations. This leads to a more complicated (Markovian) stochastic map for three ratios $\left(k^{(1)}, k^{(2)}, k^{(3)}\right)$ of conditioned partition functions, rather than just one. In spite of this we manage to prove rigorously that the physics of the two RFIM versions (sequential versus synchronous) are asymptotically identical, by first reducing the number of relevant ratios in the synchronous dynamics case down to a single ratio $k$, followed by a demonstration that double iteration of the sequential dynamics Markovian process (as derived in [2,3]) is equivalent to a single iteration of the synchronous dynamics process as derived here. This result contributes significantly to our as yet modest general knowledge of the relation between the equilibrium states induced by sequential versus parallel dynamics in Ising spin systems, which so far has been build up mostly via the study of mean-field models. We recover phases where the familiar devil's staircase form appears for the integrated densities of local magnetizations and nearest neighbour spin correlations, and we find a non-zero ground state entropy (which, due to the temperature dependence of the pseudo-Hamiltonian obeys non-standard thermodynamic relations) with an infinite number of singularities as function of the random field strength, similar to what was found earlier for sequential (bond-)disordered Ising chains in [5]. 


\section{References}

[1] Fan C and McCoy B M 1969 Phys. Rev. 182 614-23

[2] Brandt U and Gross W 1978 Z. Phys. B 31 237-45

[3] Bruinsma R and Aeppli G 1983 Phys. Rev. Lett. 50 1494-7

[4] Aeppli G and Bruinsma R 1983 Phys. Lett. A 97 117-20

[5] Derrida B, Vannimenus J and Pomeau Y 1978 J. Phys. A: Math. Gen. 11 4749-65

[6] Grinstein G and Mukamel D 1983 Phys. Rev. B 27 4503-6

[7] Gardner E and Derrida B 1985 J. Stat. Phys. 39 367-77

[8] Normand J M, Mehta M L and Orland H 1985 J. Phys. A: Math. Gen. 18 621-39

[9] Györgyi G and Ruján P 1987 J. Phys. C: Solid State Phys. 17 4207-12

[10] Luck J M, Funke M and Nieuwenhuizen Th M 1991 J. Phys. A: Math. Gen. 24 4155-96

[11] Behn U and Zagrebnov V A 1987 J. Stat. Phys. 47 939-46

[12] Evangelou S N 1987 J. Phys. C: Solid State Phys. 20 L511-9

[13] Bene J and Szépfalusy P 1988 Phys. Rev. A 37 1703-7

[14] Little W A 1974 Math. Biosci. 19 101-20

[15] Peretto P 1984 Biol. Cybern. 50 51-62

[16] Lebowitz J L, Maes C and Speer E R 1990 J. Stat. Phys. 59 117-70

[17] Sherrington D and Kirkpatrick S 1975 Phys. Rev. Lett. 35 1792-6

[18] Nishimori H 1997 TITECH Report unpublished

[19] Amit D J, Gutfreund H and Sompolinsky H 1985 Phys. Rev. Lett. 55 1530-3

[20] Fontanari J F and Köberle R 1988 J. Physique 49 13-23

[21] Skantzos N S PhD Thesis in preparation

[22] Lieb E and Mattis D (ed) 1966 Mathematical Physics in One Dimension (New York: Academic) p 123 